\title{
Perianal Disease Onset Age is Associated with Need for Abdominal Surgery in Perianal Crohn's Disease: A Ten-year Observational Study in China
}

Haichao Wang

Shanghai Tenth People's Hospital

Yaling Wu

Shanghai Tenth People's Hospital

Chen Ye

Soochow University

Zhanju Liu

Shanghai Tenth People's Hospital

Xiaolei Wang ( $\nabla$ wangxiaolei@tongji.edu.cn )

Shanghai Tenth People's Hospital

Research Article

Keywords: Perianal disease, Crohn's disease, paediatric onset, adult-onset, abdominal surgery

Posted Date: February 1st, 2021

DOl: https://doi.org/10.21203/rs.3.rs-154364/v1

License: (9) This work is licensed under a Creative Commons Attribution 4.0 International License.

Read Full License 


\section{Abstract}

Background and aims: The significance of different ages of perianal disease (PD) onset in patients with perianal Crohn's disease (PCD) remains unknown. We aimed to investigate the impact of paediatric-onset PD (POP, $<18$ years) and adult-onset PD (AOP, $\geq 18$ years) on the Crohn's disease (CD) course in a Chinese cohort.

Methods: The medical records of the diagnosed PCD patients from 2008 to 2018 were reviewed retrospectively. The cumulative incidence of abdominal surgery was calculated using the Kaplan-Meier, and independent predictors of surgery were identified by logistic regression analysis.

Results: Complex perianal fistulas $(71.7 \%$ vs $50.0 \%, p=0.011)$ and infliximab (IFX) treatment $(33.3 \%$ vs $22.0 \%, p=0.044)$ were more common among the POP patients $(\mathrm{n}=84)$. A younger PD onset age $(15.1 \pm 2.9$ vs $30.2 \pm 10.5$ years, $p<0.001)$ and shorter PCD diagnostic delay (12 vs 24 months, $p=0.033$ ) was seen in the POP cohort. AOP patients $(\mathrm{n}=209)$ had a higher rate of current smoking $(12.9 \%$ vs $4.8 \%, p=0.040)$, stricturing behaviour $(42.1 \%$ vs $27.4 \%, p=0.024)$ and abdominal surgery $(21.1 \%$ vs $4.8 \%, p=0.001)$. The cumulative probability of abdominal surgery in AOP patients was higher than that in POP patients $(p=0.007)$. In multivariable analysis, AOP (odds ratio [OR]: $2.915,95 \% \mathrm{Cl}: 1.479-5.744, p=0.002$ ) and stricturing behaviour (OR: 8.374, 95\% Cl: 1.055-66.488, $p=0.044$ ) were predictive factors for CD-related abdominal surgery in all PCD patients, complex perianal fistula was associated with the increased incidence of abdominal surgery in the AOP cohort (OR: 2.257, 95\% Cl: 1.041-4.891, $p=0.039$ ). The IFX administration significantly decreased the rate of abdominal surgery in AOP patients $(r=-0.900, p=0.037)$.

Conclusions: This Chinese cohort suggested that AOP indicate a later disease course and a higher risk of abdominal surgery than POP. Early diagnosis and positive IFX treatment could improve the prognosis of CD patients with AOP.

\section{Introduction}

Crohn's disease (CD) is a multifactorial systemic inflammatory bowel disease (IBD) with a heterogeneous clinical course. Perianal disease (PD) is a common complication in CD patients and can lead to a low quality of life, a high rate of surgical interventions, and challenging therapeutic situations [1-3]. Patients with perianal Crohn's disease (PCD) undergo a number of perianal surgical interventions (PSIs), resulting in multiple disabilities. However, the successful development of various anti-tumour necrosis factor (TNF)- $a$ agents (the first biological agents for CD in the world) breaks this impasse. Due to their rapid efficacy in cases of complicated PD and luminal lesions, anti-TNF agents, including infliximab (IFX) and adalimumab, are recommended as the first-line treatment for CD patients who have high-risk factors for poor outcomes, including PD, onset age $<40$ years, and extensive small bowel involvement [4-10]. In China, IFX was the only available anti-TNF agent for CD from 2008 to 2018 and showed a more favourable perianal and luminal outcomes than traditional treatment in both children and adult PCD patients $[11,12]$. There is some evidence that the prevalence of PCD was higher in Asian than Western 
countries, and the outcomes of Asian PCD patients may differ from those of Western patients [13]. However, Chinese studies evaluating the characteristics and outcomes of PCD patients are limited.

Patients with PCD usually have a higher risk of abdominal surgery. However, this increased risk only reached statistical significance in patients who developed PD during follow-up, but not in patients who presented with PD before or at luminal CD diagnosis[1]. This finding indicates that the PD onset time plays an important role in the CD natural history. Approximately $25 \%$ of $C D$ patients are diagnosed in childhood or adolescence [14]. Interestingly, different features have been found between paediatric- and adult-onset $C D$ patients. For example, paediatric $C D$ patients had a three-times higher risk of progressing to complicated $C D$ than adult-onset $C D$ patients [15] and required more intensive pharmacological treatments [15-17]. However, Schoepfer, A., et al. reported that adult CD patients present more bowel surgery and longer diagnostic delay (the time interval between the first symptom and diagnosis of CD) compared with paediatric $C D$ patients. And a long diagnostic delay was found to be predictive for an increased incidence of CD-related surgery only in the adult but not in the paediatric CD patients [16]. The diagnosis of perianal fistulas after the luminal CD was reported to range from $5-21 \%$ in adult $C D$ patients and $8-27 \%$ in paediatric $C D$ patients [4-7]. These suggest that onset-age plays an important role in the $C D$ disease course. However, whether the onset-age of $P D$ is associated with the incidence of CD-related abdominal surgery is still unknown. Therefore, we aimed to evaluate the measurable differences in clinical outcomes between PCD patients with paediatric-onset PD (POP) and adult-onset PD (AOP) and to identify clinical predictive factors of the development of abdominal surgery in this ten-year follow-up study.

\section{Materials And Methods}

\subsection{Patients}

We retrospectively evaluated 747 consecutive CD patients in the Tenth People's Hospital affiliated with Tongji University (Shanghai, China) from January 2008 to December 2017. Among them, 293 CD patients with perianal involvement were included and analysed in the study. Patients who were lost to follow-up or had incomplete clinical information were excluded. The follow-up period was calculated from the date of CD diagnosis to the end of data collection (December 31,2018). The study protocol was reviewed and approved by the Institutional Ethics Committee of the Tenth People's Hospital affiliated with Tongji University, Shanghai, China (SHSY- IEC- 4.0/19-23/01), and got an exemption from informed consent because of the retrospective study design.

\subsection{Description of variables and outcomes}

The medical records were reviewed for sex, PD onset age, PD type, age at CD diagnosis, age at PCD diagnosis, PCD diagnostic delay, clinical manifestations, Montreal classification, smoking status, family history of IBD, CD-related medications and surgical interventions (perianal and abdominal interventions). CD-related medical treatment included antibiotics (imidazole and quinolones), immunosuppressants (azathioprine and methotrexate), corticosteroids and IFX. IFX was regularly administered intravenously at 
a dose of $5 \mathrm{mg} / \mathrm{kg}$ body weight at 0,2 , and 6 weeks and then every 8 weeks. The duration of IFX treatment was calculated from the first infusion to the last infusion within the follow-up period. The PSIs included perianal abscess incision and drainage, seton insertion, fistulectomy, de-functioning surgery, and proctectomy. CD-related abdominal surgery referred to CD-related intestinal resections. PCD diagnostic delay was defined as the time interval between the first appearance of PD until PCD diagnosis was made.

POP was defined as PD with an onset age of less than $18(<18)$ years, and AOP was defined as PD with an onset age of greater than or equal to $18(\geq 18)$ years [16]. The diagnosis of $C D$ in paediatric patients was based on the Porto criteria [18], and the diagnosis of CD in adult patients satisfied the criteria published by the European Crohn's and Colitis Organization [19]. PD was diagnosed according to perianal symptoms, the clinical examination results together with the results of perianal ultrasound and/or magnetic resonance imaging. The types of PD were classified according to a technical review by the American Gastroenterology Association at the time of PD diagnosis and included perianal skin lesions (anal skin tags and haemorrhoids), anal canal lesions (anal fissures, ulcers, and anorectal strictures), perianal abscesses, perianal fistulas, rectovaginal fistulas, and cancer [20]. Perianal fistulas were classified into simple and complex fistulas. Simple perianal fistulas are low in position (superficial, low intersphincteric or low transsphincteric origin of the fistula tract) and have only a single external opening without evidence to suggest perianal abscesses, anorectal strictures or rectovaginal fistulas. Complex perianal fistulas originate from a high position (intersphincteric, transsphincteric, extrasphincteric, or suprasphincteric region) and usually have multiple external openings combined with perianal abscesses, anorectal structures, or rectovaginal fistulas [20].

The outcomes of PD were categorized as clinical remission, non-remission and recurrence. Clinical remission was defined as follows: (1) no perianal symptoms (e.g., local swelling and pain, haematochezia, faecal incontinence); (2) closure of the external opening of perianal fistulas; (3) no visible internal openings, perianal abscesses or anal ulcers; and (4) absence of drainage despite gentle finger compression. Non-remission referred failure to achieve clinical remission after a certain therapeutic strategy or maintain clinical remission for less than 3 months. Recurrence was defined as the presence of new PD symptoms after 3 months of clinical remission.

\subsection{Statistical analyses}

Categorical variables are described as counts with percentages and were assessed by the chi-square test or Fisher's exact test. Continuous variables are described as the mean \pm standard deviation (SD) or median plus interquartile range (IQR) and were analysed by the independent sample t-test or the MannWhitney test. The factors associated with CD-related surgery were analysed by univariate and multivariate analyses. Multivariate logistic regression analysis was performed for factors with a $p$ value $<$ 0.2 in the univariate analysis by the enter method (Wald test used for assessing $p$ values). Kaplan-Meier analysis was applied to calculate the cumulative probabilities of IFX treatment and CD-related abdominal surgery during the 10-year follow-up period. A log-rank comparison was used to compare the cumulative probabilities between the two groups of patients with POP and AOP. Spearman correlation coefficients were calculated between the rates of CD-related abdominal surgery and the percentages of patients 
treated with IFX. Two-sided $p$ values $<0.05$ were considered statistically significant. SPSS 22.0 software (IBM, Sommers, NY, USA) was used for statistical analysis.

\section{Results}

\subsection{Characteristics of PCD patients with POP and AOP}

Among 747 well-characterized CD patients, 293 (39.2\%) patients with perianal involvement were enrolled. The median follow-up duration among the 293 PCD patients was 72 (IQR: 36-108) months. Perianal fistula was the most common PCD (163/293, 55.6\%), with complex phenotypes in $57.1 \%(93 / 163)$ of cases. One hundred forty-one (48.1\%) PCD patients had perianal abscesses, and 51 (17.4\%) had haemorrhoids. Anal ulcers and fissures were seen in only 5.1\% (15/293) and 4.4\% (13/293) of PCD patients, respectively. No cases of cancer were identified during follow-up. The mean onset age of PD was $25.9 \pm 11.3$ years old. The AOP cohort comprised 209 (71.3\%) patients, and the POP cohort included 84 cases $(28.7 \%)$. There was a gradual increase in the diagnosis of POP in PCD patients from 2008.1 to 2017.12 but a reduction in the diagnosis of patients with AOP during the same period (Fig. 1).

The baseline characteristics of these two groups of patients were summarized in Table 1. Most POP (81.0\%) and AOP (73.7\%) patients were male. Among POP patients, the mean age at diagnosis of CD $(18.0 \pm 4.3$ vs $32.0 \pm 10.9$ years, $p<0.001)$ and at onset of PD $(15.1 \pm 2.9$ vs $30.2 \pm 10.5$ years, $p<0.001)$ was significantly younger than that among AOP patients (Table 1). The median duration of PCD diagnostic delay (12 [IQR: 2-48] vs 24 [IQR: 2.5-60] months, $p=0.033$ ) was shorter in the POP cohort. Compared to AOP patients, POP patients presented with a higher proportion of complex perianal fistulas (71.7\% vs 50.0\%, $p=0.011$ ) and non-stricturing and non-penetrating (B1) behaviour $(66.7 \%$ vs $47.8 \%, p=$ 0.005). Stricturing type (B2) behaviour ( $42.1 \%$ vs $27.4 \%, p=0.024)$ and current smoking $(12.9 \%$ vs $4.8 \%$, $p=0.040$ ) were more common among AOP patients than POP patients (Table 1). Two hundred forty-six (83.1\%) PCD patients achieved clinical remission of PD after a certain treatment, including 71 POP patients (84.5\%) and 175 AOP patients (83.7\%). Of the 246 patients who achieved clinical remission, 60 (24.4\%) patients experienced PD recurrence during the follow-up period, including $21(29.6 \%, 21 / 71)$ POP patients and $39(22.3 \%, 39 / 175)$ AOP patients. No significant difference in the disease location, PSI frequency, PSI type or family history of IBD was observed between the two groups of patients $(p>0.05$, Table 1). 
Table 1

Clinical characteristics of PCD patients with paediatric-onset and adult-onset perianal disease

\begin{tabular}{|c|c|c|c|}
\hline Factors & $\begin{array}{l}\text { Patients with } \\
\text { POP }(\mathrm{N}=84)\end{array}$ & $\begin{array}{l}\text { Patients with AOP } \\
(\mathrm{N}=209)\end{array}$ & $P$-value \\
\hline Male, n (\%) & $68(81.0)$ & 154 (73.7) & 0.228 \\
\hline $\begin{array}{l}\text { Mean age at } C D \text { diagnosis (years } \pm \\
\text { SD) }\end{array}$ & $18.0 \pm 4.3$ & $32.0 \pm 10.9$ & $<0.01$ \\
\hline PCD diagnostic delay (months) & $12(2-48)$ & $24(2.5-60)$ & 0.033 \\
\hline Disease location, n (\%) & $19(22.6)$ & $55(26.3)$ & 0.198 \\
\hline -L1, terminal ileum & $17(20.2)$ & $62(29.7)$ & \\
\hline -L2, colon & $45(53.6)$ & $87(41.6)$ & \\
\hline -L3, ileocolon & $3(3.6)$ & $5(2.4)$ & \\
\hline \multicolumn{4}{|l|}{-L4, upper gastrointestinal tract } \\
\hline Disease behaviour, n (\%) & $56(66.7)$ & $100(47.8)$ & \multirow{4}{*}{$\begin{array}{l}0.0150 .005^{\mathrm{a}} \\
0.024^{\mathrm{a}} 0.364^{\mathrm{a}}\end{array}$} \\
\hline -B1, non-stricturing, non-penetrating & $23(27.4)$ & $88(42.1)$ & \\
\hline -B2, stricturing & $5(6.0)$ & $21(10.0)$ & \\
\hline \multicolumn{3}{|l|}{-B3, penetrating } & \\
\hline Mean PD onset age (years $\pm S D$ ) & $15.1 \pm 2.9$ & $30.2 \pm 10.5$ & $<0.01$ \\
\hline Perianal fistula, $\mathrm{n}(\%)$ & $53(63.1)$ & $110(52.6)$ & 0.119 \\
\hline -Complex perianal fistula, n/N (\%) & $38 / 53(71.7)$ & $55 / 110(50.0)$ & 0.011 \\
\hline Perianal abscess, n (\%) & $41(48.8)$ & $100(47.8)$ & 0.898 \\
\hline Current smokers, n (\%) & $4(4.8)$ & 27 (12.9) & 0.040 \\
\hline Family history of IBD, n (\%) & $4(4.8))$ & $22(10.5)$ & 0.117 \\
\hline Medications, n (\%) & $28(33.3)$ & $46(22.0)$ & 0.044 \\
\hline - IFX & $5(6.0)$ & $13(6.2)$ & 0.925 \\
\hline - Immunomodulators & $51(60.7)$ & $128(61.2)$ & 0.933 \\
\hline - Antibiotics & $3(3.6)$ & $7(3.3)$ & 1.000 \\
\hline - Corticosteroids & & & \\
\hline
\end{tabular}

* $C D$, Crohn's disease; PCD, perianal Crohn's disease; PD, perianal disease; POP, paediatric-onset perianal disease; AOP, adult-onset perianal disease, SD, standard deviation; IQR, interquartile range; PSIs, perianal surgical interventions; IFX, infliximab; IBD, inflammatory bowel disease. ${ }^{a}$ This was a subgroup analysis of three disease behaviours between the AOP and POP groups. 


\begin{tabular}{|c|c|c|c|}
\hline Factors & $\begin{array}{l}\text { Patients with } \\
\text { POP }(\mathrm{N}=84)\end{array}$ & $\begin{array}{l}\text { Patients with AOP } \\
(\mathrm{N}=209)\end{array}$ & $P$-value \\
\hline $\begin{array}{l}\text { Time from PCD diagnosis to first use } \\
\text { of IFX, months (IQR) }\end{array}$ & $1.0(0.1-1.9)$ & $1.8(5.3-38.6)$ & $<0.01$ \\
\hline Numbers of IFX uses (mean \pm SD) & $7.7 \pm 0.75$ & $7.1 \pm 0.6$ & 0.472 \\
\hline History of PSIs, n (\%) & $66(78.6)$ & $150(71.8)$ & 0.245 \\
\hline Frequency of PSIs, n (\%) & $46(54.8)$ & $118(56.5)$ & 0.796 \\
\hline-1 time & $20(23.8)$ & $32(15.3)$ & 0.093 \\
\hline \multicolumn{4}{|l|}{ - >1 time } \\
\hline \multicolumn{4}{|l|}{ Types of perianal surgery, n (\%) } \\
\hline -Incision and drainage & 33 (39.3) & $68(32.5)$ & 0.272 \\
\hline -Seton insertion & $42(50.0)$ & $83(39.7)$ & 0.107 \\
\hline -Fistulectomy & $19(22.6)$ & $52(24.9)$ & 0.764 \\
\hline -Proctectomy & $0(0)$ & $2(1.0)$ & 1.000 \\
\hline -De-functioning & $1(1.2)$ & $2(1.0)$ & 1.000 \\
\hline History of abdominal surgery, $\mathrm{n}(\%)$ & $4(4.8)$ & $44(21.1)$ & 0.001 \\
\hline \multicolumn{4}{|c|}{$\begin{array}{l}\text { * CD, Crohn's disease; PCD, perianal Crohn's disease; PD, perianal disease; POP, paediatric-onset } \\
\text { perianal disease; AOP, adult-onset perianal disease, SD, standard deviation; IQR, interquartile range; } \\
\text { PSIs, perianal surgical interventions; IFX, infliximab; IBD, inflammatory bowel disease. }{ }^{\text {a }} \text { This was a } \\
\text { subgroup analysis of three disease behaviours between the AOP and POP groups. }\end{array}$} \\
\hline
\end{tabular}

\subsection{IFX treatment}

Seventy-four patients $(25.5 \%, 74 / 293)$ received IFX treatment after the first diagnosis of PCD in our study. The average number of IFX infusions was $7.3 \pm 0.5$. Thirty patients received IFX monotherapy, thirty-siX patients received IFX within one month after a PSI, and only eight patients were treated with both IFX and immunosuppressants.

The proportion of patients treated with IFX was significantly higher in the POP group $(33.3 \%$ vs $22.0 \%, p=$ 0.044 , Table 1). The median time from diagnosis of PD to the first infusion of IFX was shorter among POP patients than AOP patients (1 month, IQR: $0.1-1.9$ vs 22 months, IQR: $5.3-38.6, p<0.01$, Table 1 ). The cumulative probability of IFX therapy at $1,3,5$ and 10 years was $69.1 \%, 77.6 \%, 79.5 \%$ and $90.4 \%$ in POP patients versus $43.4 \%, 53.7 \%, 57.8 \%$ and $84.7 \%$ in AOP patients, respectively $(p<0.001$, Fig. $2 A)$. No significant differences were detected at numbers of IFX infusion between patients with POP and AOP ( $p>$ 0.05 , Table 1).

\subsection{Surgical treatment}


Forty-eight patients (17.4\%) suffered CD-related abdominal surgery during the follow-up period. The percentage of patients who underwent surgery was significantly higher in the AOP cohort $(21.1 \%$ vs $4.8 \%$, $p=0.001$, Table 1). Furthermore, the cumulative probability of CD-related abdominal surgery after CD diagnosis was also higher in AOP patients $(9.0 \%$ at 1 year, $12.1 \%$ at 3 years, $18.4 \%$ at 5 years, and $31.8 \%$ at 10 years) than in POP patients (2.7\% at 1 year, $2.7 \%$ at 3 years, $2.7 \%$ at 5 years, and $8.5 \%$ at 10 years, $p$ $=0.007$, Fig. $2 \mathrm{~B}$ ).

In the multivariate logistic analysis (Table 2), AOP (OR: $2.915,95 \% \mathrm{Cl}: 1.479-5.744, p=0.002$ ) and stricturing behaviour (B2 vs B3, OR: 8.374, 95\% Cl: 1.055-66.488, $p=0.044$ ) were associated with a higher risk of CD-related abdominal surgery among the 293 PCD patients. Other factors, including sex, age at CD diagnosis, PCD diagnostic delay, current smoking status, PD type and family history of IBD, were not associated with CD-related abdominal surgery $(p>0.05)$. Furthermore, AOP patients with complex perianal fistulas were more likely to undergo CD-related abdominal surgery (OR: $2.257,95 \% \mathrm{Cl}$ : $1.041-4.891, p=0.039$, Table 2). However, no factor was found to predict CD-related abdominal surgery in POP patients ( $p>0.05$, Table 2$)$. 
Table 2

Factors associated with the risk of abdominal surgery

\begin{tabular}{|c|c|c|c|c|}
\hline \multirow[t]{2}{*}{ Factors } & \multicolumn{2}{|l|}{ Univariate analysis } & \multicolumn{2}{|l|}{ Multivariate analysis } \\
\hline & OR $(95 \% \mathrm{Cl})$ & P-value & OR $(95 \% \mathrm{Cl})$ & P-value \\
\hline \multicolumn{5}{|l|}{ All PCD patients $(n=293)$} \\
\hline Male & $1.727(0.883-3.379)$ & 0.110 & $1.380(0.683-2.788)$ & 0.370 \\
\hline AOP & $2.887(1.511-5.515)$ & 0.001 & $2.915(1.479-5.744)$ & 0.002 \\
\hline Behaviour & $1.805(0.957-3.402)$ & 0.049 & $5.656(0.707-45.279)$ & 0.090 \\
\hline$-\mathrm{B} 1$ vs B3 & $0.245(0.032-1.905)$ & 0.068 & $8.374(1.055-66.488)$ & 0.103 \\
\hline -B2 vs B3 & & 0.179 & & 0.044 \\
\hline IFX + PSIs & $0.270(0.063-1.163)$ & 0.079 & $0.251(0.057-1.110)$ & 0.068 \\
\hline \multicolumn{5}{|l|}{ Patients with AOP $(n=209)$} \\
\hline PCD diagnostic delay & $0.944(0.986-1.001)$ & 0.109 & $0.994(0.986-1.001)$ & 0.109 \\
\hline Behaviour & $3.488(0.435-27.976)$ & 0.061 & $3.595(0.440-29.390)$ & 0.089 \\
\hline$-\mathrm{B} 1 v s \mathrm{~B} 3$ & $6.769(0.858-53.416)$ & 0.239 & $6.563(0.817-52.714)$ & 0.233 \\
\hline -B2 vs B3 & & 0.070 & & 0.077 \\
\hline IFX + PSIs & $0.324(0.073-1.433)$ & 0.138 & $0.374(0.082-1.712)$ & 0.205 \\
\hline Complex perianal fistulas & $2.297(1.089-4.848)$ & 0.029 & $2.257(1.041-4.891)$ & 0.039 \\
\hline \multicolumn{5}{|l|}{ Patients with POP $(n=84)$} \\
\hline Family history of IBD & $4.102(0.861-19.532)$ & 0.076 & - & - \\
\hline \multicolumn{5}{|c|}{$\begin{array}{l}\text { * CD, Crohn's disease; PD, perianal disease; L1, terminal ileum; L2, colon; L3, ileocolon; L4, upper } \\
\text { gastrointestinal tract; B1, non-stricturing and non-penetrating; B2, stricturing; B3, penetrating; IFX, } \\
\text { infliximab; PSIs, perianal surgical interventions; PCD, perianal Crohn's disease; AOP, adult-onset } \\
\text { perianal disease; POP, paediatric-onset perianal disease; IBD, inflammatory bowel disease. }\end{array}$} \\
\hline
\end{tabular}

From 2008.1 to 2017.12, the proportion of patients receiving IFX treatment increased in both the POP and AOP groups (Fig. 3). The occurrence of CD-related abdominal surgery showed a gradually decreasing trend in AOP patients (Fig. 3A). And a negative correlation was found between the proportion of patients receiving IFX treatment and undergoing abdominal surgery only in AOP patients $(r=-0.900, p=0.037)$ but not in POP patients $(r=0.667, p=0.219)$.

\section{Discussion}


The presence of PD in CD patients usually indicates a distinct and aggressive disease course $[2,21,22]$. There is some evidence that the prevalence of PCD was higher in Asian than Western countries, and the outcomes of Asian PCD patients may differ from those of Western patients [13]. The increasing tendency in China over the past few decades calls for more attention to be paid to the natural course of PCD in these newly developed disease population. However, Chinese studies evaluating the characteristics and outcomes of PCD patients are limited, and the significance of PD development at different ages is unclear. This is the first comparative study to investigate this issue among a Chinese 10-year observational cohort study in the first years of the biologic treatment era. Thus, this study allowed us to obtain key updated knowledge about the differences between PCD patients with paediatric and adultonset PD concerning the need for abdominal surgery. Firstly, we found that CD patients who developed PD during childhood had a more serious perianal phenotype and earlier PCD diagnosis and IFX treatment, while more aggressive luminal disease, current smoking and a higher rate of abdominal surgery were more common in AOP patients. Secondly, AOP and stricturing luminal behaviour were associated with an increased incidence of abdominal surgery. Finally, IFX administration could decrease the rate of abdominal surgery in AOP patients. These results indicate that more attention should be paid to patients with AOP and highlight the important role of early diagnosis and IFX therapy in achieving better clinical outcomes.

Previous studies have shown that approximately one-quarter of CD patients are diagnosed in childhood [14]. Paediatric CD patients usually show distinct features, which are characterized by more severe disease activity and biological therapy than adult CD cases $[15,23]$. In our study, $28.7 \%$ of PCD patients developed their first perianal symptoms during the paediatric period, and they presented with more complex perianal fistulas. However, the stricturing phenotype of luminal CD and CD-related abdominal surgery were more common in AOP patients. Thus, early-onset (paediatric-onset) PD presents more complicated local manifestations of PD, but late-onset (adult-onset) PD is more prone to progress to severe luminal damage progression. These results not only indicate distinct clinical characteristics and monitoring focus in CD patients who develop PD at different ages but also suggest the presence of distinct disease patterns for perianal and luminal CD.

Moreover, we found that PCD patients with POP had earlier use of IFX and a higher cumulative risk of IFX treatment. In the past decade, IFX was the only available biological agent for Chinese CD patients [24]. Due to its efficacy in treating complicated CD, IFX has been recommended widespreadly in cases of earlyonset or fistulizing PCD as a top-down treatment strategy in recent years [4-10]. We found that POP has been increasing over time, which means there is a trend for POP patients to be diagnosed and treated more recently. PCD diagnosis was performed earlier in POP patients than AOP patients. Therefore, the higher percentage of complex perianal fistulas, early-onset and recent diagnosis of PD in the POP group may contribute to the more frequent and earlier use of IFX.

The presence of PD speeds up the complexity of the natural history of $C D$, accelerates the development of luminal stenosis, and increases the risk of abdominal surgery in some studies of the pre-biologic era [2, 21, 22]. However, most of these studies focused on the adult-onset cohort, they neglected the influence of 
age of the PD development and biological agents on the CD outcomes. Recently, a Korean study reported that the presence of PD at CD diagnosis did not increase the risk for abdominal surgical intervention [13]. And a Danish population-based study found that the occurrence of PD at CD diagnosis was not predictive of the risk for undergoing intestinal resection, whereas the development of PD during follow-up was [1]. These results raise the possibility that the impact of the PD development time on the intestinal resection of $\mathrm{CD}$ patients may differ. The later the PD developed, the more common the complicated luminal damage and abdominal surgery presented. This result is inconsistent with the previous conclusion that the early-onset CD was associated with an increased incidence of CD-related surgery [25]. But our results are similar to a recent study. They showed that there were more intestinal strictures and abdominal operations in adult CD patients than in paediatric CD patients, which was related to the longer diagnostic delay in adults [16]. Similarly, we found the patient with AOP (late-onset PD) presented a higher rate of structuring behaviour and abdominal surgery. Factors including AOP and stricturing behaviour were predictive for increased CD-related abdominal surgery among all PCD patients. The use of IFX treatment also showed a negative correlation with the rate of abdominal surgery in patients with AOP. This verified the conclusion that the increased use of biologics is positively correlated with a reduced need for abdominal surgery [26]. Furthermore, we observed that the time from the onset of PD to the diagnosis of PCD and the time from the diagnosis of PCD to the first use of IFX were longer in the adult-onset cohort. These results all suggest a later disease course and later IFX treatment in AOP patients. It has become increasingly apparent that the early use of IFX can decrease the occurrence of CD-related surgery in adult CD $[17,27]$. Therefore, the more complicated luminal disease, underuse of IFX treatment and delayed initiation of IFX may contribute to the higher rate of abdominal surgery in AOP patients.

In addition, smoking has been reported as a definite factor associated with recurrence after surgery and a poor response to medical therapy in CD patients [28]. Thus, the greater proportion of current smokers also explained the higher rate of abdominal surgery in AOP patients. These findings indicate that a close monitoring of luminal lesions and a positive therapeutic strategy, including the early and extensive use of IFX treatment and cessation of smoking, should be undertaken in PCD patients with AOP to reduce the rate of abdominal surgery.

One of the strengths of our study is the inclusion of all kinds of PCD, leading to a reduction in selection bias. The different clinical characteristics and outcomes of PCD patients with POP and AOP were directly compared in this study. We focused on the influence of PD onset age on the rate of abdominal surgery in CD patients, and found that there was an higher risk of abdominal surgery in PCD patients who developed PD at adults. These findings should alert physicians to pay close attention to these patients to ensure that they receive intensive treatment and close monitoring to achieve better outcomes in clinical practice.

However, there are some limitations to our study. The first limitation is the retrospective and single-centre design. Secondly, all of the PCD patients included in our study were hospitalized patients, who may have had relatively serious manifestations. Lastly, because of the nature of the general hospital, we only receive patients over 14 years old, so we cannot investigate the clinical characteristics and outcomes of 
CD patients with very early onset PD. And no factor was found to predict CD-related abdominal surgery in POP patients. Therefore, prospective, large-scale and multi-centre or population-based studies are required.

In summary, the age of PD development in PCD patients plays an important role in clinical prognoses. PCD patients with AOP showed a delay PCD diagnosis, underuse of IFX treatment and a higher risk for abdominal surgery than those with POP. These results suggest that the clinical impact of PD on the luminal outcomes of $C D$ patients may be less prominent in patients with POP than those with AOP in this Chinese IBD centre. Early diagnosis and IFX treatment of CD in patients with AOP may therefore allow for earlier identification and management of potential CD-related luminal damage and reduce the incidence of abdominal surgery.

\section{Abbreviations}

$C D$, Crohn's disease; PD, perianal disease; $P C D$, perianal Crohn's disease; IBD, inflammatory bowel disease; POP, paediatric-onset PD; AOP, adult-onset PD; TNF, tumour necrosis factor; IFX, infliximab; PSIs, perianal surgical interventions.

\section{Declarations}

\section{Ethics approval and consent to participate:}

The study complied with the Helsinki Declaration, and the study protocol and exemption of informed consent were approved by the Institutional Ethics Committee of the Tenth People's Hospital of Tongji University, Shanghai, China (SHSY- IEC- 4.0/19-23/01).

\section{Consent for publication:}

Not applicable.

\section{Availability of data and materials:}

The datasets used and analysed during the current study available from the corresponding author on reasonable request.

\section{Competing interests:}

The authors declare that the research was conducted in the absence of any commercial or financial relationship that could be construed as a potential conflict of interest. 


\section{Funding:}

This study was supported by a grant from the National Natural Science Foundation of China (grant number 81970449).

\section{Author Contributions:}

Haichao Wang, Chen Ye and Xiaolei Wang designed this study. Haichao Wang, Chen Ye, and Yaling Wu collected and interpreted the data. Haichao Wang and Yaling Wu analysed the data. All authors contributed to drafting the article. Zhanju Liu was responsible for critically revising the article. Xiaolei Wang was responsible for all the data and results in this study and the eventual revision of the entire paper. All authors have approved the final version of this manuscript.

\section{Acknowledgements:}

The authors thank all patients and colleagues of Shanghai Tenth People's Hospital for their support.

\section{Authors' information:}

${ }^{1}$ Department of Gastroenterology, Shanghai Tenth People's Hospital, Tongji University, Shanghai 200072, China. ${ }^{2}$ Medical College of Soochow University, Suzhou 215000, Jiangsu Province, China

\section{References}

1. Zhao, M., et al., A 10-Year Follow-up Study of the Natural History of Perianal Crohn's Disease in a Danish Population-Based Inception Cohort. Inflamm Bowel Dis, 2019. 25(7): p. 1227-1236. doi: 10.1093/ibd/izy374

2. Yoon, J.Y., et al., Effects of Perianal Involvement on Clinical Outcomes in Crohn's Disease over 10 Years. Gut Liver, 2018. 12(3): p. 297-305. doi: 10.5009/gnl17275

3. Moon, C.M., et al., Clinical features and predictors of clinical outcomes in Korean patients with Crohn's disease: a Korean association for the study of intestinal diseases multicenter study. J Gastroenterol Hepatol, 2014. 29(1): p. 74-82. doi: 10.1111/jgh.12369

4. Keljo, D.J., et al., Course and treatment of perianal disease in children newly diagnosed with Crohn's disease. Inflamm Bowel Dis, 2009. 15(3): p. 383-7. doi: 10.1002/ibd.20767

5. Gupta, N., et al., Incidence of stricturing and penetrating complications of Crohn's disease diagnosed in pediatric patients. Inflamm Bowel Dis, 2010. 16(4): p. 638-44. doi: 10.1002/ibd.21099

6. Singer, A.A., et al., Fistulizing Crohn's Disease Presenting After Surgery on a Perianal Lesion. Pediatrics, 2016. 137(3): p. e20152878. doi: 10.1542/peds.2015-2878 
7. Vernier-Massouille, G., et al., Natural history of pediatric Crohn's disease: a population-based cohort study. Gastroenterology, 2008. 135(4): p. 1106-13. doi: 10.1053/j.gastro.2008.06.079

8. Panaccione, R., et al., Review article: treatment algorithms to maximize remission and minimize corticosteroid dependence in patients with inflammatory bowel disease. Aliment Pharmacol Ther, 2008. 28(6): p. 674-88. doi: 10.1111/j.1365-2036.2008.03753.x

9. Danese, S., et al., Review article: infliximab for Crohn's disease treatment-shifting therapeutic strategies after 10 years of clinical experience. Aliment Pharmacol Ther, 2011. 33(8): p. 857-69. doi: 10.1111/j.1365-2036.2011.04598.x

10. Loly, C., J. Belaiche, and E. Louis, Predictors of severe Crohn's disease. Scand J Gastroenterol, 2008. 43(8): p. 948-54. doi: 10.1080/00365520801957149

11. Dupont-Lucas, C., et al., Predictors of response to infliximab in paediatric perianal Crohn's disease. Aliment Pharmacol Ther, 2014. 40(8): p. 917-29. doi: 10.1111/apt.12928

12. Sands, B.E., et al., Infliximab maintenance therapy for fistulizing Crohn's disease. N Engl J Med, 2004. 350(9): p. 876-85. doi: 10.1056/NEJMoa030815

13. Song, E.M., et al., Clinical outcomes and long-term prognosis of perianal Crohn's disease in an Asian population. J Gastroenterol Hepatol, 2020. doi: 10.1111/jgh.15308

14. Henderson, P., et al., The changing epidemiology of paediatric inflammatory bowel disease. Aliment Pharmacol Ther, 2011. 33(12): p. 1380-1; author reply 1381-2. doi: 10.1111/j.13652036.2011.04660.x

15. Pigneur, B., et al., Natural history of Crohn's disease: comparison between childhood-and adult-onset disease. Inflamm Bowel Dis, 2010. 16(6): p. 953-61. doi: 10.1002/ibd.21152

16. Schoepfer, A., et al., Systematic Analysis of the Impact of Diagnostic Delay on Bowel Damage in Paediatric Versus Adult Onset Crohn's Disease. J Crohns Colitis, 2019. 13(10): p. 1334-1342. doi: 10.1093/ecco-jcc/jjz065

17. Herman, Y., et al., The Characteristics and Long-term Outcomes of Pediatric Crohn's Disease Patients with Perianal Disease. Inflamm Bowel Dis, 2017. 23(9): p. 1659-1665. doi: 10.1097/MIB.0000000000001171

18. Ibd Working Group of the European Society for Paediatric Gastroenterology, H. and Nutrition, Inflammatory bowel disease in children and adolescents: recommendations for diagnosis-the Porto criteria. J Pediatr Gastroenterol Nutr, 2005. 41(1): p. 1-7. doi: 10.1097/01.mpg.0000163736.30261.82

19. Gomollon, F., et al., 3rd European Evidence-based Consensus on the Diagnosis and Management of Crohn's Disease 2016: Part 1: Diagnosis and Medical Management. J Crohns Colitis, 2017. 11(1): p. 3-25. doi: 10.1093/ecco-jcc/jjw168

20. Sandborn, W.J., et al., AGA technical review on perianal Crohn's disease. Gastroenterology, 2003. 125(5): p. 1508-30. doi: 10.1016/j.gastro.2003.08.025

21. Chun, J., et al., Association of Perianal Fistulas with Clinical Features and Prognosis of Crohn's Disease in Korea: Results from the CONNECT Study. Gut Liver, 2018. 12(5): p. 544-554. doi: $10.5009 / \mathrm{gnl} 18157$ 
22. Xavier, S., et al., Perianal Crohn's disease - association with significant inflammatory activity in proximal small bowel segments. Scand J Gastroenterol, 2018. 53(4): p. 426-429. doi:

10.1080/00365521.2018.1437928

23. Paul, T., et al., Distinct phenotype of early childhood inflammatory bowel disease. J Clin Gastroenterol, 2006. 40(7): p. 583-6. doi: 10.1097/00004836-200608000-00004

24. Park, E.J., et al., The efficacy of infliximab combined with surgical treatment of fistulizing perianal Crohn's disease: Comparative analysis according to fistula subtypes. Asian J Surg, 2018. 41(5): p. 438-447. doi: 10.1016/j.asjsur.2017.06.005

25. Romberg-Camps, M.J., et al., Influence of phenotype at diagnosis and of other potential prognostic factors on the course of inflammatory bowel disease. Am J Gastroenterol, 2009. 104(2): p. 371-83. doi: 10.1038/ajg.2008.38

26. Brunet, E., et al., Time Trends of Crohn's Disease in Catalonia from 2011 to 2017. Increasing Use of Biologics Correlates with a Reduced Need for Surgery. J Clin Med, 2020. 9(9). doi: 10.3390/jcm9092896

27. Thia, K.T., et al., Risk factors associated with progression to intestinal complications of Crohn's disease in a population-based cohort. Gastroenterology, 2010. 139(4): p. 1147-55. doi: 10.1053/j.gastro.2010.06.070

28. Parkes, G.C., K. Whelan, and J.O. Lindsay, Smoking in inflammatory bowel disease: impact on disease course and insights into the aetiology of its effect. J Crohns Colitis, 2014. 8(8): p. 717-25. doi: 10.1016/j.crohns.2014.02.002

\section{Figures}




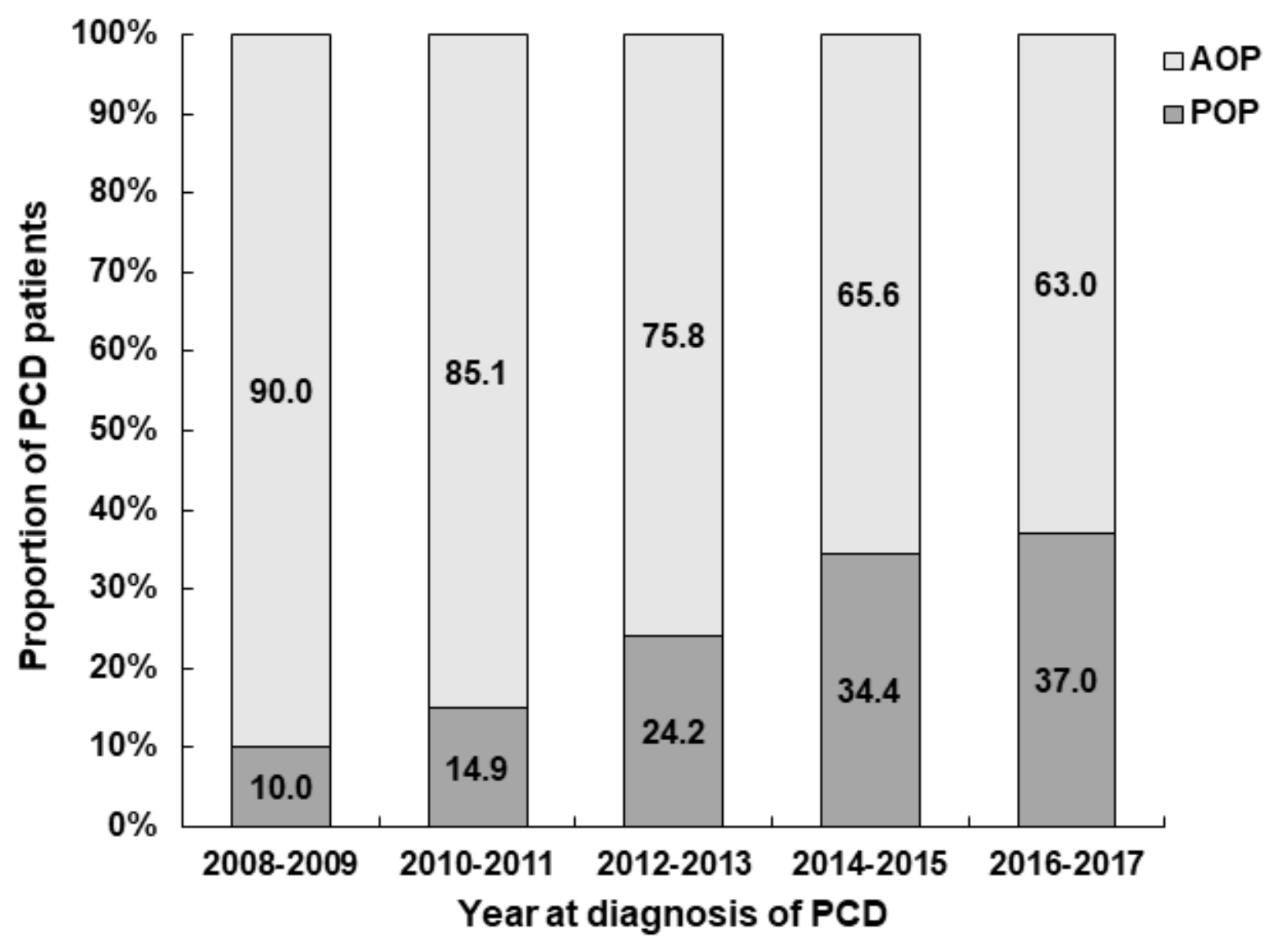

Figure 1

Proportion of PCD patients with different perianal disease onset ages between 2008 and 2017. PCD, perianal Crohn's disease; POP, paediatric-onset perianal disease; AOP, adult-onset perianal disease.
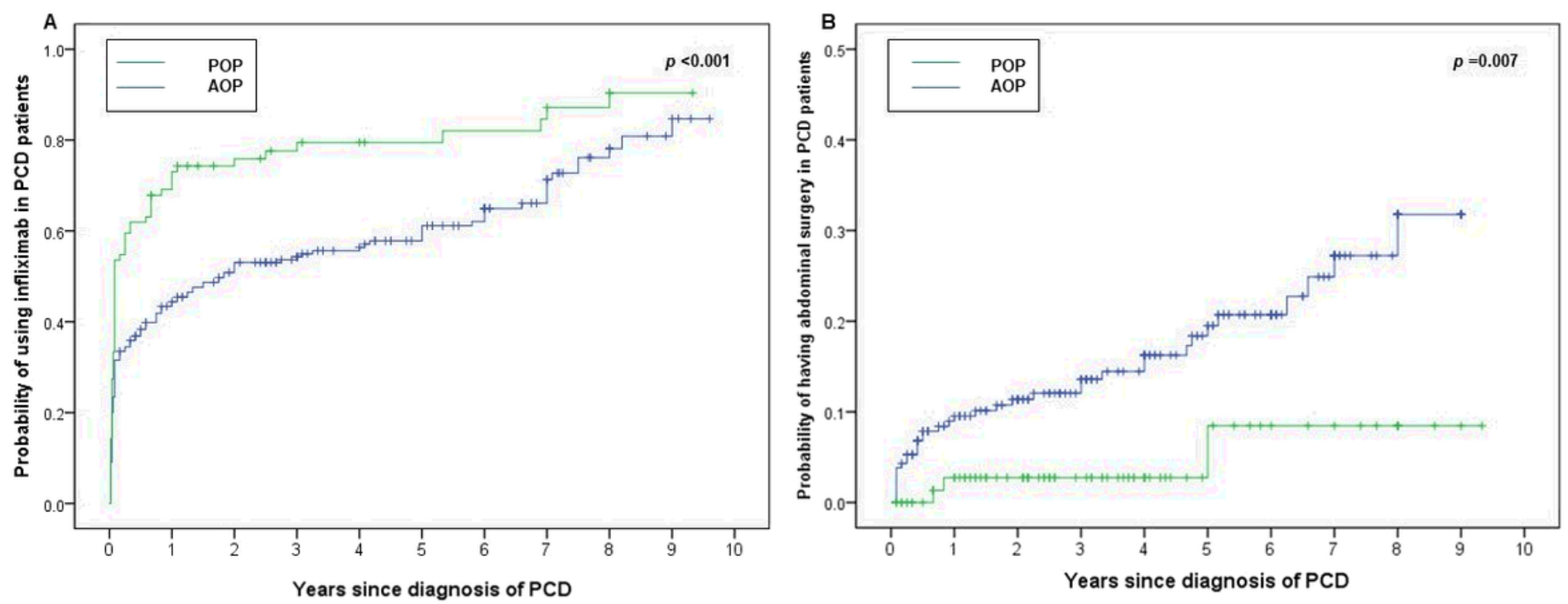

Figure 2 
Cumulative probability of (A) infliximab and (B) CD-related abdominal surgery in patients with paediatriconset and adult-onset perianal disease. PCD, perianal Crohn's disease; POP, paediatric-onset perianal disease; AOP, adult-onset perianal disease.
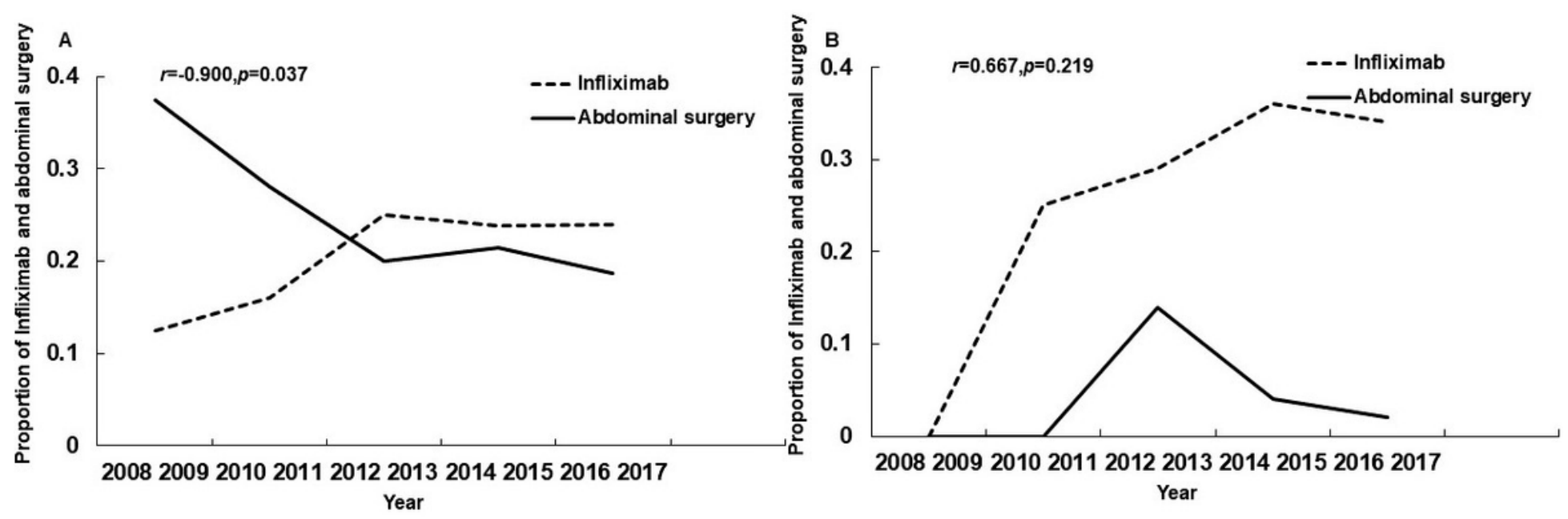

\section{Figure 3}

Ten-year trend and correlation between infliximab and CD-related abdominal surgery in CD patients with (A) adult-onset and (B) paediatric-onset perianal disease. 